\title{
Uterine rupture in early pregnancy
}

Keywords: uterine rupture, trial of labor after cesarean delivery, early pregnancy, cesarean scar pregnancy

\section{Introduction}

Uterine rupture is a life-threatening obstetrical complication which incidence has been increasing. This condition usually occurs in the setting of trial of labor after cesarean delivery and few reports have described its occurrence in early pregnancy. We report a case of an uterine rupture in the thirteenth week of pregnancy, in a woman with two previous cesarean sections and a short inter pregnancy interval. We highlight the most important differential diagnosis, therapeutic approaches and future obstetric implications. Routine screening of cesarean section scars in early pregnancy must be considered, since this is a major risk factor leading to early uterine rupture.

Uterine rupture consists of a total disruption of uterine layers and it is a life-threatening obstetrical complication for both mother and fetus. ${ }^{1}$ A previous transmyometrial surgical incision is the single most important risk factor for this condition, being the rupture of an unscarred uterus a very rare event (about 1 in 15000$)^{2,3}$ The incidence of rupture in scarred uteri has been increasing in the last few years, probably reflecting the increasing cesarean section rates in most resource-rich countries, which now exceed $20 \% .{ }^{4,5}$ The overall incidence of uterine rupture in women with a previous cesarean section varies between 0.3 to 1 percent. ${ }^{6}$ Almost all uterine ruptures in developed countries occur in the third trimester of pregnancy, near term and, mainly, in the setting of trial of labor after cesarean delivery (TOLAC). ${ }^{7}$ Poor Bishop score on admission to labor, labor induction with prostaglandins (mainly misoprostol) and labor dystocia have been stablished as important risk factors that affect the incidence of uterine rupture during TOLAC. ${ }^{7}$ Advanced maternal and gestational age, high parity, macrossomia, short inter-delivery interval $(<18$ to 24 months) and single-layer uterine closure were also associated with increased risk for uterine rupture.6 For this last risk factor, there are some conflicting results. ${ }^{8}$ First and second trimester uterine ruptures are very unusual. ${ }^{9,10}$ The first report of a spontaneous early pregnancy scar rupture was published in 1982 in Denmark. ${ }^{11}$ Although early uterine rupture is associated with high maternal and fetal morbidity and mortality, there are few case reports of conservative managment of the uterine defect with successful outcome.,12 Clinical signs of uterine rupture in early pregnancy must be distinguished from other acute abdominal and obstetric emergencies. The most relevant differential diagnosis is ectopic pregnancy, although bleeding from corpus luteum, miscarriage, heterotropic pregnancy and molar pregnancy with secondary invasion can be considered. ${ }^{4,9}$ Abdominal pain, vaginal bleeding and vomiting are common findings in uterine rupture and emergent surgical intervention is generally needed since intraabdominal hemorrhage can lead to progressive maternal hemodynamic deterioration. ${ }^{9}$ Generally, the uterine defect occurs in the previous incision and it is closed like a traditional hysterotomy closure with additional interventions, as needed, to ensure hemostasis. If that is not possible, a hysterectomy may be performed. ${ }^{5}$ Considering its rarity, here we report a case of an early pregnancy uterine rupture.
Volume 2 Issue 6 - 2017

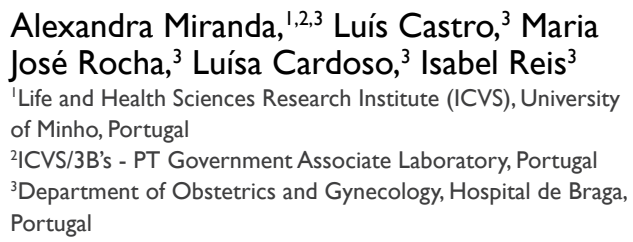

Correspondence: Alexandra Miranda, Life and Health Sciences Research Institute (ICVS), School of Medicine, University of Minho, Campus Gualtar, 47। 0-057 Braga, Portugal, Tel +35। 916738596,Email alexandramir@gmail.com

Received: August 21, 2017 | Published: October 25, 2017

\section{Case report}

A 32-year-old woman, with personal history of obesity and previous gastric sleeve surgery (actual BMI of $41 \mathrm{Kg} / \mathrm{m}^{2}$ ), was admitted to our emergency department complaining of generalized abdominal pain and vaginal bleeding that started few hours earlier and progressively got worse. She was otherwise healthy, having had two previous cesarean sections, both with two-layer hysterotomy closure, the first one for breech presentation and the second for suspected dystocia, about 10 months before. She also mentioned irregular menses, a 13-week amenorrhea period and a positive pregnancy test two days before. On physical examination, the vital signs were stable (blood pressure $123 / 82 \mathrm{mmHg}$, pulse rate 86 beats per minute) and the abdomen was tender, particularly in lower quadrants. On gynecological examination, blood clots and, apparently, products of conception were seen coming through the external os. The cervix was partially opened and uterine mobilization caused severe pain. Our first impression was that it was likely to be a miscarriage. Transvaginal ultrasound examination revealed a thickened endometrium and an extra-uterine gestational sac with a 13 -week consistent, fetal heartbeat negative fetus, next to the anterior wall of the uterus (Figure 1). Further inspection showed, in the same localization, a heterogeneous nodular structure likely to be a hematoma or placental tissue. A considerable amount of fluid was also observed in the Douglas pouch. Haemoglobin concentration was $12,9 \mathrm{~g} / \mathrm{dL}$. Our preoperative diagnosis was then uterine rupture. Laparotomy confirmed a moderate haemoperitoneum and showed fetus and placenta in the abdominal cavity next to the uterine rupture that occurred throughout the extent of the previous hysterorrhaphy (Figure 2). The products of conception were removed (Figure 3) and a double-layer uterine repair was performed with vicryll suture. In the first post-operative day, blood tests showed a haemoglobin concentration of $9 \mathrm{~g} / \mathrm{dL}$. The patient's postoperative recovery was otherwise uneventful and she was discharged on her third postoperative day with a prescription of iron tablets. On the first post-operative month appointment the patient reported a surgical site infection that was treated accordingly and she had no present symptoms. As the patient had decided against tubal ligation, even 
after being advised about the risks of a subsequent pregnancy, she was counseled about contraception and was also recommended to undergo an elective cesarean section in future pregnancies. She opted by a levonorgestrel intrauterine system insertion.

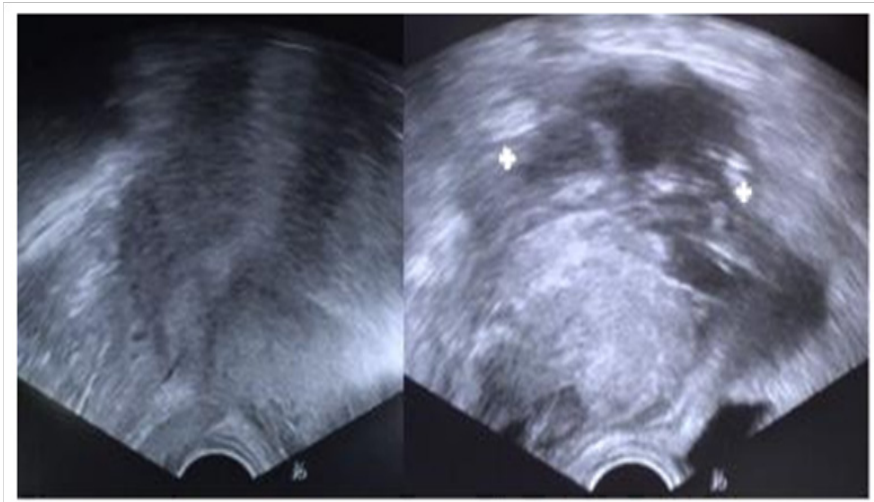

Figure I Transvaginal ultrasound showing a thickened endometrium (left) and an extra-uterine gestational sac with a I3-week consistent, fetal heartbeat negative fetus (calipers), in the vesicouterine pouch, with a heterogeneous nodular structure next to it (right).

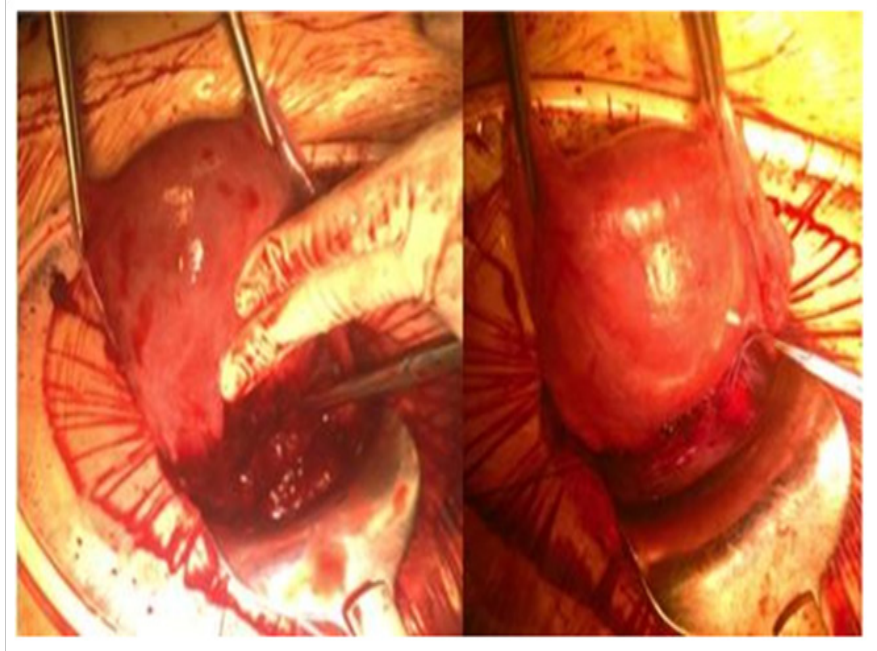

Figure 2 Uterine rupture throughout the extent of the previous hysterorrhaphy (left) and uterine defect closure (right).

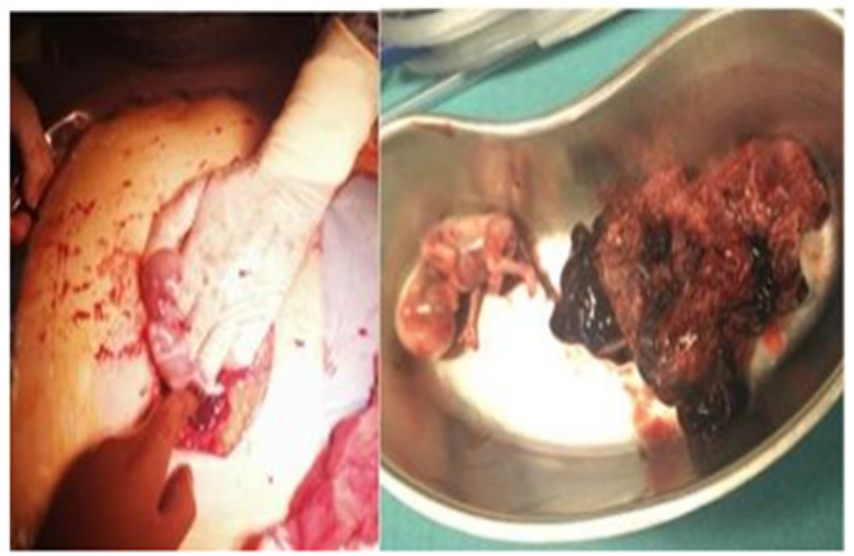

Figure 3 Fetus and placental tissue found in the abdominal cavity.

\section{Discussion}

Uterine rupture consists of an obstetrical emergency that is becoming more frequent in developed countries, perhaps as a consequence of increasing cesarean section rates. ${ }^{4}$ Although the majority of cases occur in late gestation, mainly during TOLAC, very few reports have described its occurrence in the first and second trimesters of pregnancy, whether in scarred but also (rarely) in unscarred uteri. ${ }^{9,10,13}$ Besides cesarean section, hysteroscopic resection of uterine septum, myomectomy, and cornual resection or iatrogenic uterine perforation were described as other risk factors for early uterine rupture. Other less common causes include multiparity, placenta increta, congenital anomalies, trauma and medical assisted pregnancy with embryo transfer. ${ }^{14}$ Here, we report a case of an uterine rupture in the thirteenth week of pregnancy, in a woman with two recognizable risk factors: two previous cesarean sections and a short interpregnancy interval. Considering the moderate vaginal bleeding and the externalization of apparent products of conception, active miscarriage was the first suspected differential diagnosis. Nevertheless, given the severe pelvic pain, whether spontaneous or after uterine mobilizations, and the abdominal tenderness, rupture of an ectopic pregnancy was not to exclude. The transvaginal scan confirmed the presence of a gestacional sac in the vesico-uterine pouch but, concerning fetal biometries, consistent with a thirteenth week nonviable fetus, the hypothesis of an ectopic pregnancy was unlikely, since the patient had been asymptomatic until that time. Given the facts, the possibility of a uterine rupture was then raised as the most likely diagnosis and it was confirmed during laparotomy. A particular complication of a pregnancy after cesarean delivery is a cesarean scar pregnancy, which consists in the implantation of the gestational sac in the myometrium of the previous histerotomy scar. ${ }^{15,16}$ The incidence of this condition is extremely low (about $0,05 \%$ among women with a prior cesarean delivery) and the most frequent symptom is painless vaginal bleeding. ${ }^{4,16}$ Ultrasonographic findings of a gestational sac at the site of previous myometrial scar in the presence of an empty uterine cavity and cervical canal, with a thin myometrium adjacent to the bladder, makes the diagnosis. ${ }^{16,17}$ The high-velocity, low-impedance, turbulent flow at the implantation area in a thin uterine scar makes the risk for uterine rupture with dangerous internal bleeding very high, as pregnancy progresses. ${ }^{16}$ In the case reported, this event was a differential diagnosis but the definite diagnosis was not possible, as there was not previous imaging of the current pregnancy. Nevertheless, according to G Sliutz et al. ${ }^{15}$ all first trimester uterine ruptures are caused by scar implantation of the trophoblast. ${ }^{15}$ Prevention of this obstetric condition will depend on rotine screening of cesarean section scars in an early gestational stage and the avoidance of unnecessary elective cesarean sections. Concerning treatment of uterine rupture, the aims of conservative surgery are to repair de uterine defect, controlling the hemorrhage and additional damage to other organs (mainly urinary tract). ${ }^{4}$ Standard repair technique has not been established. Hysterectomy should be considered based upon patient's request to preserve fertility, the extent of damage and surgeon's skills and patient's clinical stability. ${ }^{4}$ This procedure was reported in 14 to 33percent of women with a uterine rupture. ${ }^{18}$ Bilateral tubal ligation is also advised given the recurrence risk of uterine ruptures in subsequent pregnancies, which is estimated to be between 4 and $19 \% .{ }^{9,19}$ Therefore, if the woman wishes to preserve fertility, counseling must highlight the need to undergo a cesarean section in all future pregnancies. Recurrent rupture can 
occur early in pregnancy and is unpredictable. ${ }^{20}$ There is no consensus on the ideal timing for delivery, but an elective cesarean delivery at 37 weeks of gestation without prior testing of fetal lung maturity seems a reasonable approach. ${ }^{4}$ In conclusion, early pregnancy uterine rupture is a rare and potentially life threatening event which incidence is increasing given the actual high cesarean section rate. Clinical signs of this condition are nonspecific and must be distinguished from other acute abdominal emergencies and other obstetric events. Cesarean scar pregnancy must be considered as a major risk factor leading to early uterine rupture.

\section{Acknowledgements}

There has been no significant financial support for this work that could have influenced its outcome. We confirm that the manuscript has been read and approved by all named authors and that there are no other persons who satisfied the criteria for authorship but are not listed. We further confirm that the order of authors listed in the manuscript has been approved by both of us. We confirm that we have given due consideration to the protection of intellectual property associated with this work and that there are no impediments to publication.

\section{Conflict of interest}

We wish to confirm that there are no known conflicts of interest associated with this publication.

\section{References}

1. Suner S, Jagminas L, Peipert J, et al. Fatal spontaneous rupture of a gravid uterus: Case report and literature review of uterine rupture. J Emerg Med. 1996;14(2):181-185.

2. Turner MJ. Uterine rupture. Best Pract Res Clin Obstet Gynaecol. 2002;16(1):69-79.

3. Sallam AH, Preston J. Idiopathic uterine perforation in late pregnancy. $J$ Obstet Gynaecol. 2002;22(3):317.

4. http://www.uptodate.com/contents/uterine-rupture-after-previous-cesarean-delivery

5. Al-Zirqi I, Stray-Pedersen B, Forsén L, et al. Uterine rupture: trends over 40years. BJOG. 2015;123(5):780-787.

6. Porreco RP, Clark SL, Belfort MA, et al. The changing specter of uterine rupture. Am J Obstet Gynecol. 2009;200(3):269.e1-269.e4.
7. Hofmeyr GJ, Say L, Gülmezoglu AM. WHO systematic review of maternal mortality and morbidity: the prevalence of uterine rupture. BJOG. 2005;112:1221-1228.

8. The CAESAR study collaborative group. Caesarean section surgical techniques: a randomised factorial trial (CAESAR). BJOG. 2010;117(11):1366-1376.

9. Tola EN. First Trimester Spontaneous Uterine Rupture in a Young Woman with Uterine Anomaly. Case Reports in Obstetrics and Gynecology. 2014;2014:967386.

10. Ijaz S, Mahendru A, Sanderson D. Spontaneous uterine rupture during the 1st trimester: a rare but life-threatening emergency. Journal of Obstetrics and Gynaecology. 2011;31(8):772.

11. Helkjaer PE, Petersen PL. Rupture of the uterus in the 11th week of pregnancy. Ugeskr Laeger. 1982;144:3836-3837.

12. Naim NM, Ahmad S, Siraj HH, et al. Advanced abdominal pregnancy resulting from late uterine rupture. Obstet Gynecol. 2008;111(2 Pt 2):502-504.

13. http://www.uptodate.com/contents/rupture-of-the-unscarred-uterus

14. Cunningham Williams J. Williams Obstetrics. New York: McGraw-Hill Medical; 2010.

15. Sliutz G, Sanani R, Spängler-Wierrani B, et al. First trimester uterine rupture and scar pregnancy. Med Hypotheses. 2009;73(3):326-327.

16. Ash A, Smith A, Maxwell D. Caesarean scar pregnancy. BJOG. 2007;114(3):253-263.

17. Rotas M, Haberman S, Levgur M. Cesarean Scar Ectopic Pregnancies. Obstet Gynecol. 2006;107(6):1373-1381.

18. National Institutes of Health Consensus Development Conference Panel. National Institutes of Health Consensus Development conference statement: vaginal birth after cesarean: new insights. Obstet Gynecol. 2010;115(6):1279-1295.

19. Ahmadi S, Nouira S, Bibi S, et al. Uterine rupture of the unscarred uterus. About 28 cases. Gynecologie, Obstetrique \& Fertilite. 2003;31(9):713-717.

20. Chibber R, El-Saleh E, Al Fadhli R, et al.Uterine rupture and subsequent pregnancy outcome- how safe is it? A 25-year study. J Matern Fetal Neonatal Med. 2010;23(5):421-424. 